\title{
Sarcoidosis Uveitis Mimicking the Presentation of Intraocular Lymphoma
}

\author{
Jeremy G. Light ${ }^{\mathrm{a}}$, John R. Chancellor ${ }^{\mathrm{b}}$, Sami H. Uwaydat ${ }^{\mathrm{b}}$, Nikhil Meena ${ }^{\mathrm{c}}$, \\ Chien Chen ${ }^{\mathrm{d}}$, Ahmed B. Sallam ${ }^{\mathrm{b}, \mathrm{e}}$
}

\begin{abstract}
We aimed to report a case of sarcoidosis presenting with atypical clinical findings that mimicked intraocular lymphoma. A 73-year-old female presented with flashes and decreased vision in her left eye over a 2 -week period. The patient had no previous ocular medical history. Initial fundal examination revealed vitritis (vitreous inflammation) with retinal hemorrhage and subretinal yellowish lesions in the left eye. All tests were negative for infectious causes. Serum angiotensin-converting enzyme (ACE) was normal. Flow cytometric analysis of vitreous showed a suspicious $\mathrm{CD}^{+} \mathrm{T}$ lymphoid population missing $\mathrm{CD} 7$ surface antigen and an elevated $\mathrm{CD} 4 / \mathrm{CD} 8$ ratio. Chest $\mathrm{CT}$ showed hilar lymphadenopathy. Clinical findings to this point raised the suspicion of lymphoma. Hilar lymph node biopsy demonstrated granulomatous inflammation, confirming the diagnosis of sarcoidosis. Sarcoidosis presenting with uveitis in an elderly patient may pose a diagnostic challenge due to variable presentation and absence of diagnostic criteria. Retinitis and subretinal infiltrates can be a misleading clinical finding of sarcoidosis masquerading as an intraocular oncologic process such as lymphoma.
\end{abstract}

Keywords: Sarcoidosis; Intraocular lymphoma; Subretinal infiltrates

\section{Introduction}

Sarcoidosis is a multisystem disorder with variable clinical manifestations including ocular inflammation. The most frequent lo-

Manuscript submitted October 8, 2018, accepted October 22, 2018

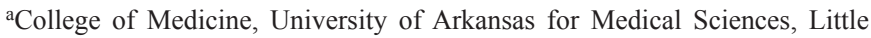
Rock, AR, USA

bJones Eye Institute, University of Arkansas for Medical Sciences, Little Rock, AR, USA

${ }^{\mathrm{c}}$ Winthrop P. Rockefeller Cancer Institute, University of Arkansas for Medical Sciences, Little Rock, AR, USA

dDepartment of Pathology, University of Arkansas for Medical Sciences, Little Rock, AR, USA

'Corresponding Author: Ahmed Sallam, Jones Eye Institute, University of Arkansas for Medical Sciences, 4301 W. Markham St., Little Rock, AR 72205, USA. Email: ahmedsallam11@yahoo.com

doi: https://doi.org/10.14740/jmc3190 cation of sarcoid ocular inflammation is the anterior segment [1]. Sarcoid associated posterior uveitis commonly presents with inflammatory choroidal nodules, optic nerve granulomas or retinal vasculitis $[1,2]$. Definitive diagnosis of sarcoidosis is made upon detection of noncaseating granuloma via biopsy.

Posterior segment inflammation has an extensive differential diagnosis including infectious causes, immunologic conditions and malignancies such as lymphomas. Intraocular lymphoma is a malignant form of non-Hodgkin lymphoma that presents as chronic vitritis with subretinal infiltrates refractory to corticosteroid therapy $[3,4]$. Herein, we present an unusual case of sarcoid associated posterior uveitis presenting with retinitis and subretinal yellowish infiltrative lesions.

\section{Case Report}

A 73-year-old Caucasian woman was referred to our institution by her community retina specialist for evaluation of abnormal retinal lesions. There was no significant past ocular history. She had a medical history of non-insulin dependent type 2 diabetes and hypertension. She reported weight loss, profuse sweating, and loss of appetite over the last 3 weeks. Family history was remarkable of lymphoma in the sister.

On examination, visual acuity was $20 / 25$ in the right eye and 20/30-2 in the left eye. Slit lamp examination revealed trace vitreous inflammation in the right and $3+$ in the left. Anterior segment was non-revealing in both eyes with no sign of inflammatory corneal deposits (keratic precipitates) or anterior chamber reaction. Initial fundal examination revealed mild inferior retinal hemorrhage with no definite infiltrates in the right eye. In the left eye there were subretinal yellowish infiltrative lesions and hemorrhage up to the inferior arcade with some healed retinal lesions in the periphery seen through the vitritis (Fig. 1a).

Fluorescein angiography (FA) revealed mild disc staining in the right eye. There was significant leakage from the disc and inferior vessels in the left eye (Fig. 1b). Optical coherence tomography (OCT) of the left eye showed foveal outer retinal band elevation and disruption with a hyperintense subretinal infiltrate (Fig. 1c). Indocyanin green angiography showed dilated inferior choroidal vessels in the left eye. Serum testing was negative for Treponema pallidum and Toxoplasma antibodies. Angiotensin-converting enzyme (ACE) and serum calcium levels were normal. T-SPOT tuberculosis test was 


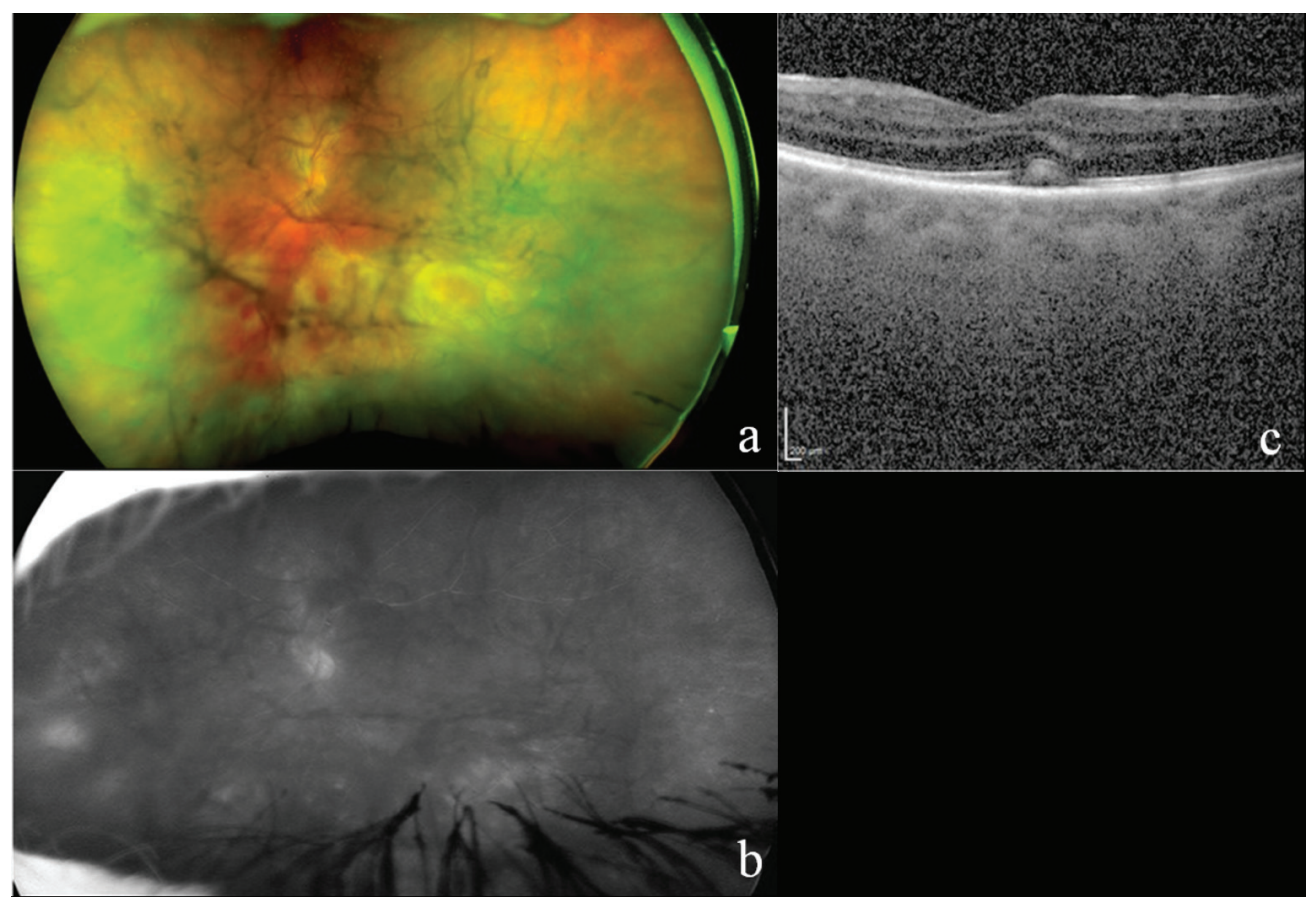

Figure 1. (a) Color fundus photograph with hemorrhage and yellowish subretinal infiltrates present up to the inferior arcade in the left eye with dense vitritis and vitreous haze. (b) Fluorescein angiography demonstrating pronounced leakage from disc and inferior vessels in the left eye despite a hazy view of the fundus. (c) Optical coherence tomography revealing indistinct choroidal details, foveal outer retinal band elevation and disruption in the left eye, with a hyperintense subretinal infiltrate in addition to a mild epiretinal membrane.

negative. Chest X-ray and subsequent chest CT with contrast revealed hilar lymphadenopathy (Fig. 2a-b).

Because of the wide range of differential diagnosis in this case, we performed a pars plana vitrectomy with vitreous biopsy. Polymerase chain analysis (PCR) of the vitreous was negative for Herpes viruses and Toxoplasma gondii; bacterial and

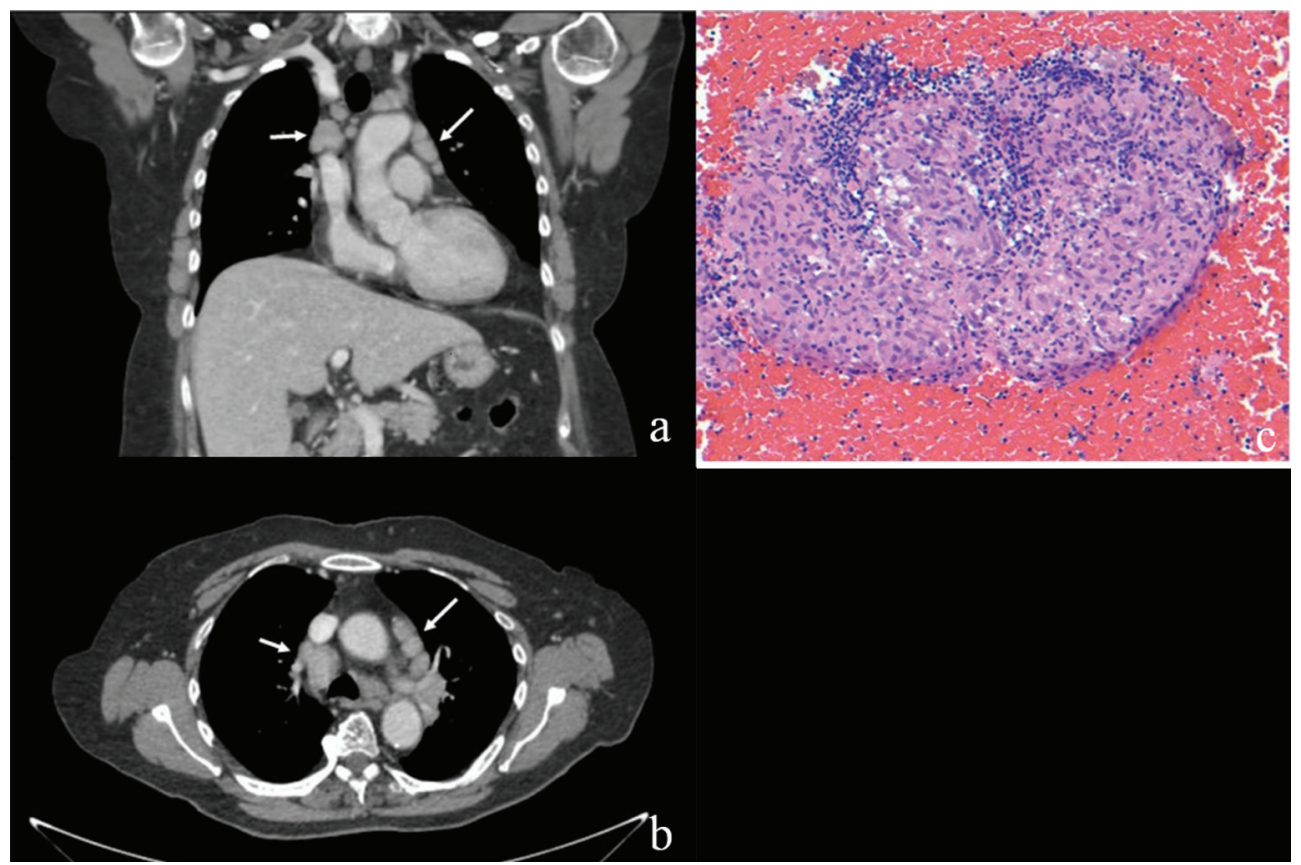

Figure 2. (a, b) Coronal and axial chest CT with contrast demonstrating bilateral hilar lymphadenopathy (white arrows). (c) Low magnification $(\times 200) \mathrm{H} \& \mathrm{E}$ stain of hilar biopsy showing extensive granulomatous inflammation with no caseation. 


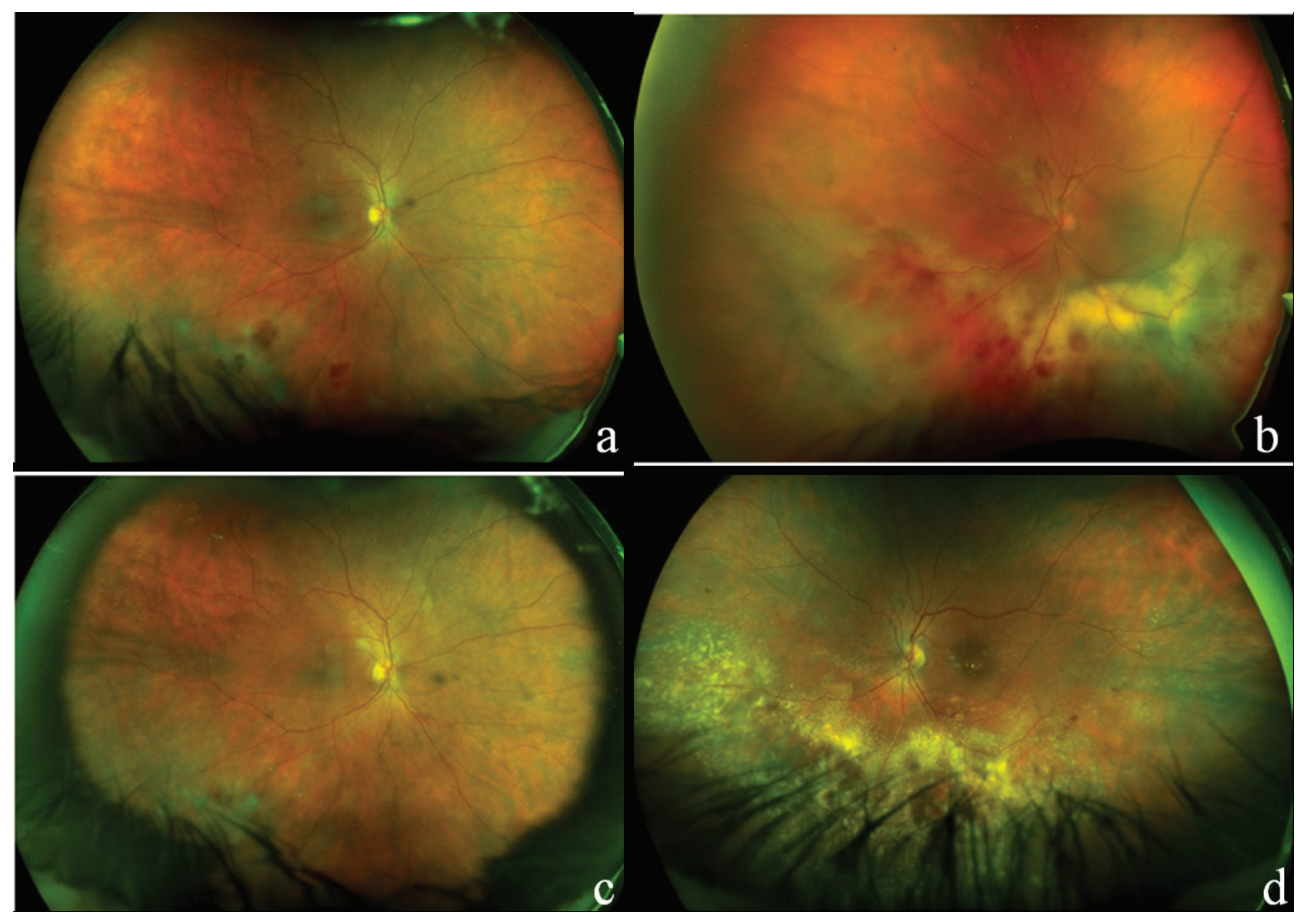

Figure 3. Fundus examination following left vitrectomy/vitreous biopsy before initiation of treatment (a, b) and after 4 months of oral prednisone treatment $(c, d)$. After treatment photograph demonstrates retinal exudates and mild hemorrhage (left more than right) and no significant vitreous haze in either eye.

fungal cultures were also negative. Histological examination of the vitreous sample showed intermediate-sized lymphoid cells with mature morphology. Flow cytometric analysis revealed CD4 T lymphoid population with a subset missing CD7 surface antigen and an elevated CD4/CD8 ratio. This raised the concern for intraocular lymphoma and prompted referral to the oncology service. Small mobile lymph nodes were palpable in the anterior cervical triangle, but lumbar puncture and CSF analysis were normal. Endobronchial ultrasound (EBUS) biopsy of hilar lymph nodes was undertaken and results were consistent with noncaseating granulomatous inflammation rather than a neoplastic process (Fig. 2c). Both the lung aspirate and lymph node biopsy staining were negative for acid fast bacilli. These findings supported a diagnosis of sarcoidosis rather than lymphoma.

The patient was started on oral prednisone initially at 60 $\mathrm{mg}$, tapering down over 6 weeks to $5 \mathrm{mg}$ every other day. A sustained-release dexamethasone intravitreal implant (Ozurdex) was also injected into the left eye. She responded well to the corticosteroid regimen with resolution of macular edema and chorioretinal inflammation in the left eye after treatment but required repeated treatment with intravitreal dexamethasone implants for both eyes throughout the follow-up period of 15 months (Fig. 3).

\section{Discussion}

Sarcoidosis is diverse in its systemic and ocular manifesta- tions. The clinical presentation of our patient was an unusual combination of retinitis with subretinal infiltrates. This atypical presentation emulated the presentation of ocular lymphoma.

The differential diagnosis in this patient at presentation included infectious causes such as viral retinitis (herpes simplex virus (HSV), cytomegalovirus (CMV), Epstein-Barr virus (EBV), varicella-zoster virus (VZV) or West Nile virus (WNV)), tuberculosis, syphilis, Lyme disease, fungal endophthalmitis, Toxoplasma chorioretinits; and inflammatory causes such as sarcoidosis and Behcet's disease in addition to intraocular lymphoma. The absence of associated systemic features such as skin and mucous membrane ulcers made the diagnosis of Behcet's disease unlikely. Blood tests and vitreous PCR tests were negative for causes of infectious retinitis. Fundus examination revealed yellowish subretinal infiltrates in her left eye, which are suggestive of intraocular lymphoma [5, $6]$. The presentation of these lesions contrasts with the posterior segment lesions of sarcoidosis which present as multifocal choroiditis, vasculitis or granulomas [1]. Vitreous biopsy was consistent with intraocular lymphoma but was not conclusive. There were no multinucleated giant cells upon cytologic examination of the vitreous fluid indicative of sarcoid inflammation. Elevation of CD4/CD8 ratio can be found in chronic inflammatory conditions as well as lymphoma [7, 8]. A final diagnosis of sarcoidosis was made based on the noncaseating granulomas found in the hilar lymph nodes.

There are two points in this case that deserve attention. First, laboratory tests and initial biopsy results were inconclusive of sarcoidosis. Serum ACE and calcium were both normal. 
In sarcoidosis patients with uveitis, sensitivity of raised serum ACE was $73 \%$ and specificity $83 \%$ [1]. Serum lysozyme was not tested in this case. When both ACE and serum lysozyme are raised, the predictive value of diagnosis of sarcoidosis is $83 \%$ [9]. Vitreous biopsy was also inconclusive of the diagnosis but concerning for lymphoma. Second, the clinical presentation was atypical for sarcoidosis [10]. There was an absence of anterior uveitis, keratic precipitates, choroidal or optic disc granulomas. Subretinal infiltrates and retinitis are unusual manifestations of sarcoid granulomatous inflammation. The clinical picture was concerning for lymphoma, including the hilar adenopathy. Ultimately, EBUS biopsy of the lymph nodes provided a definitive diagnosis of sarcoidosis.

In summary, retinitis and subretinal infiltrates can be an atypical, misleading clinical finding of sarcoidosis, masquerading as intraocular lymphoma.

\section{Conflict of Interest}

The authors declare that there is no conflict of interest regarding the publication of this article.

\section{Financial Support}

No grant or financial support received.

\section{Abbreviations}

FA: fluorescein angiography; OCT: optical coherence tomography; ACE: angiotensin-converting enzyme; PCR: polymerase chain analysis; EBUS: endobronchial ultrasound; HSV: herpes simplex virus; CMV: cytomegalovirus; EBV: EpsteinBarr virus; VZV: varicella-zoster virus; WNV: West Nile virus

\section{References}

1. Rothova A. Ocular involvement in sarcoidosis. Br J Ophthalmol. 2000;84(1):110-116.

2. Stavrou P, Linton S, Young DW, Murray PI. Clinical diagnosis of ocular sarcoidosis. Eye (Lond). 1997;11(Pt 3):365370.

3. Coupland SE, Heimann H, Bechrakis NE. Primary intraocular lymphoma: a review of the clinical, histopathological and molecular biological features. Graefes Arch Clin Exp Ophthalmol. 2004;242(11):901-913.

4. Peterson K, Gordon KB, Heinemann MH, DeAngelis LM. The clinical spectrum of ocular lymphoma. Cancer. 1993;72(3):843-849.

5. Batchelor T, Loeffler J. Clinical presentation, pathologic features, and diagnosis of primary central nervous system lymphoma. UpToDate. Post TW, 2015. http://www.uptodate.com.

6. Birnbaum AD, Huang W, Sahin O, Tessler HH, Goldstein DA. Ocular sarcoidosis misdiagnosed as primary intraocular lymphoma. Retina. 2010;30(2):310-316.

7. Davis JL, Viciana AL, Ruiz P. Diagnosis of intraocular lymphoma by flow cytometry. Am J Ophthalmol. 1997;124(3):362-372.

8. Kojima K, Maruyama K, Inaba T, Nagata K, Yasuhara T, Yoneda K, Sugita S, et al. The CD4/CD8 ratio in vitreous fluid is of high diagnostic value in sarcoidosis. Ophthalmology. 2012;119(11):2386-2392.

9. Baarsma GS, La Hey E, Glasius E, de Vries J, Kijlstra A. The predictive value of serum angiotensin converting enzyme and lysozyme levels in the diagnosis of ocular sarcoidosis. Am J Ophthalmol. 1987;104(3):211-217.

10. Herbort CP, Rao NA, Mochizuki M, members of Scientific Committee of First International Workshop on Ocular S. International criteria for the diagnosis of ocular sarcoidosis: results of the first International Workshop On Ocular Sarcoidosis (IWOS). Ocul Immunol Inflamm. 2009;17(3):160-169. 